\title{
Microspot x-ray focusing using a short focal-length compound refractive lenses
}

\author{
Y. I. Dudchik, a) N. N. Kolchevsky, and F. F. Komarov \\ Institute of Applied Physics Problems, Kurchatova 7, 220064, Minsk, Belarus
}

M. A. Piestrup, ${ }^{\text {b) }}$ J. T. Cremer, C. K. Gary, and H. Park

Adelphi Technology, Inc. 981-B Industrial Road, San Carlos, California 94070

\author{
A. M. Khounsary \\ Argonne National Laboratory, 9700 S. Cass Avenue, Argonne, Illinois 60439
}

(Received 3 March 2004; accepted 13 August 2004; published 1 November 2004)

\begin{abstract}
We have fabricated and tested short focal-length compound refractive lenses (CRLs) composed of microbubbles embedded in epoxy encased in glass capillaries. The interface between the bubbles formed 90 to 350 spherical biconcave microlenses reducing the overall focal length inversely by the number of lenses or bubbles. When compared with CRLs manufactured using other methods, the microbubble lenses have shorter focal lengths with higher transmissions and larger gains for moderate energy x rays (e.g., 7-20 keV). We used beamline 2-3 at the Stanford Synchrotron Radiation Laboratory and beamline 5BM-D-DND at the Advanced Photon Source to measure focal lengths between 100-250 mm with lens apertures varying between 97 and $321 \mu \mathrm{m}$. Transmission profiles were measured giving, for example, a peak transmission of $46 \%$ for a $240 \mathrm{~mm}$ focal length CRL at $20 \mathrm{keV}$. The focal-spot sizes were also measured yielding, for example, a vertical spot size of $1.2 \mu \mathrm{m}$ resulting from an approximate 20-fold demagnification of the APS $23 \mu \mathrm{m}$ source size. The measured gains in intensity over that of unfocused beam were between 9 and 26. (C) 2004 American Institute of Physics. [DOI: 10.1063/1.1809289]
\end{abstract}

\section{INTRODUCTION}

As is the case for visible optics, ${ }^{1}$ the focal length of a series of $N$ closely spaced identical lenses is reduced by $1 / N$. Tomie and A. Snigirev et al. showed this to be true for $\mathrm{x}$-ray wavelengths using a series of holes drilled into a single substrate with the spaces between holes forming cylindrical lenses. ${ }^{2-4}$ These cylindrical biconcave lenses form a compound refractive lens (CRL) that is capable of focusing and collimating $\mathrm{x}$ rays from 5 to $80 \mathrm{keV}$. Other CRLs were developed to obtain two-dimensional focusing that included the "coin" CRL ${ }^{5-12}$ and the "microcapillary" or "bubble" CRL. ${ }^{13-17}$ The coin CRL is a series of thin disks with a spherical or parabolic dimple embossed on each side, stacked coaxially to form the CRL. ${ }^{6-10}$

A bubble lens consists of a series of bubbles encased in an epoxy-filled glass capillary, the material between the bubbles forming a biconcave spherical lens. ${ }^{13}$ The liquid or epoxy between the two bubbles forms the biconcave lens under the action of surface tension forces. Various polymers in the form of epoxy can be used. The polymers, as a rule, consist of carbon, hydrogen, and nitrogen; each of them is characterized by a low absorption coefficient for $\mathrm{x}$ rays. The epoxy used in these experiments is $\mathrm{C}_{100} \mathrm{H}_{200} \mathrm{O}_{20} \mathrm{~N}$ and has a density of $1.08 \mathrm{~g} / \mathrm{cc}$. The method of fabrication is given in Ref. 14.

The bubble lens has five attributes that make it attractive

\footnotetext{
${ }^{\text {a)} E l e c t r o n i c ~ m a i l: ~ d u d c h i k @ b s u . b y ~}$

b)Electronic mail: melpie@adelphitech.com
}

for use in imaging and focusing: (1) Because of the nature of the physics forming the bubble, the lens surface quality is extremely good, probably much better than any other method for forming the lens, (2) the capillary "enclosure" ensures that the series of unit lenses or bubbles are extremely well aligned coaxially, (3) the minimum thickness of the lens, $d$, can be made extremely thin $(d<25 \mu \mathrm{m})$, (4) the radius of curvature can be made to be much smaller than other methods of fabrication, resulting in fewer lenses required for a

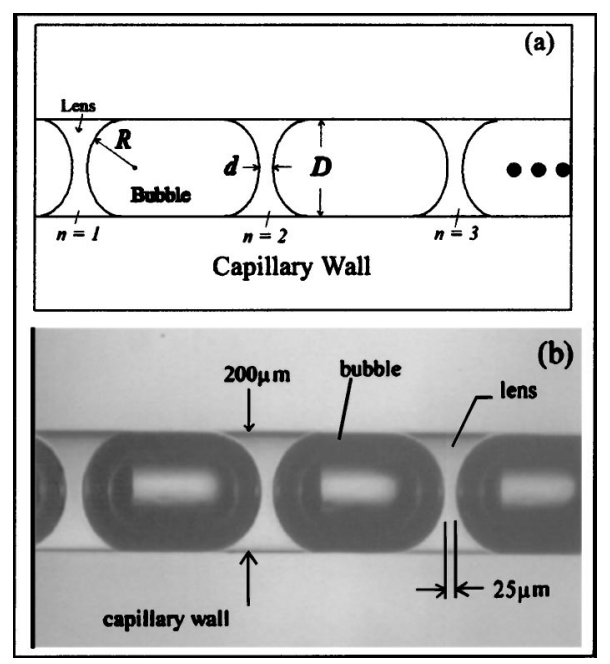

FIG. 1. (a) A diagram of a capillary filled by epoxy concave microlenses divided by air bubbles. (b) A photograph of a bubble compound refractive lens. 
TABLE I. Measured and calculated parameters of bubble CRLs for SSRL.

\begin{tabular}{|c|c|c|c|c|c|c|}
\hline Bubble lens designation & $1-1-90$ & $3-1-196$ & $3-4-103$ & $3-3-93$ & $4-1-102$ & $4-1-102$ \\
\hline Photon energy & 12 & 12 & 9 & 8 & 8 & 7 \\
\hline Number of lenses & 90 & 196 & 103 & 93 & 102 & 102 \\
\hline$R$, radius of curvature $(\mu \mathrm{m})$ & 165 & 250 & 100 & 100 & 100 & 100 \\
\hline Observed lens minimum wall thickness $(\mu \mathrm{m})$ & 76 & 41 & 37 & 36 & 23 & 36 \\
\hline Visual lens minimum wall thickness $(\mu \mathrm{m})$ & 10 & 50 & 25 & 25 & 10 & 10 \\
\hline Calculated refractive index decrement & $1.74 \mathrm{E}-06$ & $1.74 \mathrm{E}-0.6$ & $3.09 \mathrm{E}-06$ & $3.91 \mathrm{E}-06$ & $3.91 \mathrm{E}-06$ & $5.12 \mathrm{E}-06$ \\
\hline Tabulated linear attenuation coefficient $\left(\mathrm{cm}^{-1}\right)$ & 1.49 & 1.49 & 3.77 & 5.50 & 5.50 & 8.40 \\
\hline Calculated focal length $(\mathrm{cm})$ & 52.8 & 37 & 15.7 & 13.8 & 12.55 & 9.6 \\
\hline Calculated image distance $(\mathrm{cm})$ & 54.5 & 37.8 & 15.8 & 13.9 & 12.6 & 9.7 \\
\hline Measured image distance $(\mathrm{cm})$ & 32 & 36 & 17.5 & 13 & 14 & 10 \\
\hline Calculated vertical minimum waist diameter $(\mu \mathrm{m})$ & 15.1 & 10.4 & 4.4 & 3.9 & 3.2 & 2.7 \\
\hline Measured vertical minimum waist diameter $(\mu \mathrm{m})$ & 12.8 & 12 & 3.9 & 4.8 & 2.7 & 4 \\
\hline Calculated horizontal minimum waist diameter $(\mu \mathrm{m})$ & 64.1 & 44.0 & 18.8 & 16.6 & 13.5 & 11.5 \\
\hline Measured peak transmission & $36 \%$ & $30 \%$ & $24 \%$ & $16 \%$ & $27 \%$ & $5 \%$ \\
\hline Calculated attenuation aperture diameter $(\mu \mathrm{m})$ & 314 & 262 & 143 & 125 & 119 & 96.7 \\
\hline Measured attenuation aperture diameter $(\mu \mathrm{m})$ & 321 & 245 & 147 & 150 & 149 & 149 \\
\hline Calculated demagnification & 0.032 & 0.022 & 0.009 & 0.008 & 0.007 & 0.006 \\
\hline Calculated two-dimensional gain & 16.6 & 20.0 & 25.6 & 16.9 & 28.9 & 6.0 \\
\hline Measured two-dimensional gain & 8.9 & 3.5 & 13.4 & $\cdots$ & 25.5 & $\cdots$ \\
\hline
\end{tabular}

given focal length, and (5) the method and materials of fabrication result in an extremely robust and inexpensive CRL. Items 1-4 all result in a high transmission low-loss CRL. Indeed, by increasing the number of unit lenses (or bubbles), the focal length can be made much smaller than other methods. Short focal lengths result in high-gain CRLs capable of producing submicron spot sizes.

As shown in Fig. 1, a series of $N+1$ bubbles forms $N$ biconcave lenses, each of focal length $f_{1}$, resulting in a focal length $f$ of

$$
f=\frac{f_{1}}{N}=\frac{R}{2 N \delta} .
$$

The unit lens focal length $f_{1}$ is given by

$$
f_{1}=\frac{R}{2 \delta}
$$

where the complex refractive index of the unit lens material is expressed by

$$
n=1-\delta-i \beta,
$$

and $R$ is the radius of the bubble, which is equal to the radius of the capillary or $D=2 R$.
As with all CRLs, the aperture of the bubble lens is limited. This is due to absorption at the edges of the lens, making the CRL act like an iris as well as a lens. For a spherical unit lens of bubble radius $R$, the absorption aperture radius is defined by the radius, $r_{a}$, at which the image intensity is reduced by the factor $e^{-2}$, and is given by

$$
\begin{aligned}
r_{a} & =\left(\frac{2 R}{\mu N}-\frac{1}{(\mu N)^{2}}\right)^{1 / 2} \\
& \approx\left(\frac{2 R}{\mu N}\right)^{1 / 2}=\left(\frac{4 \delta f}{\mu}\right)^{1 / 2},
\end{aligned}
$$

where $\mu$ is the linear absorption coefficient of the lens material. This is the radius of the lens at which a ray passing through the lens would be attenuated by $e^{-2}$. Equation (4a) is for spherical lenses; while Eq. (4b) is for parabolic lenses. For most practical values of $N$ and $\mu$, the values for $r_{a}$ are approximately the same. The calculated value of $\mu$ for the epoxy used in the experiments is equal to $5.8 \mathrm{~cm}^{-1}$ for $8 \mathrm{keV}$

\begin{tabular}{|c|c|c|c|c|c|c|}
\hline Bubble lens designation & $6-8-178$ & $6-6-244$ & $6-1-250$ & $6-2-300$ & $6-3-349$ & $6-3-349$ \\
\hline Photon energy & 13 & 15 & 15 & 15 & 18 & 20 \\
\hline Number of lenses & 178 & 244 & 250 & 300 & 349 & 349 \\
\hline$R$, radius of curvature $(\mu \mathrm{m})$ & 100 & 100 & 100 & 100 & 100 & 100 \\
\hline Calculated focal length $(\mathrm{cm})$ & 19 & 18.5 & 18 & 15 & 18.6 & 23 \\
\hline Calculated image distance $(\mathrm{cm})$ & 19.7 & 19.1 & 18.6 & 15.4 & 19.2 & 24.0 \\
\hline Measured image distance $(\mathrm{cm})$ & 20.6 & 19.1 & 19.1 & 17 & 20.8 & 25 \\
\hline Measured vertical min. waist dia. $(\mu \mathrm{m})$ & 2.7 & 1.35 & 2.55 & 1.24 & 1.50 & 2.10 \\
\hline Measured peak transmission & $39 \%$ & $54 \%$ & $37 \%$ & $25 \%$ & $39 \%$ & $46 \%$ \\
\hline Calc. attenuation aperture dia. $(\mu \mathrm{m})$ & 198.44 & 214.8 & 211.9 & 193.5 & 242.85 & 288.96 \\
\hline Means. attenuation aperture dia. $(\mu \mathrm{m})$ & 171.5 & 176.8 & 182 & 177.5 & 191.42 & 208.93 \\
\hline Measured two-dimensional gain & 25 & 11 & $\cdots$ & 25 & $\cdots$ & $\cdots$ \\
\hline
\end{tabular}
$\mathrm{x}$ rays, and from Eq. (4b), the absorption aperture is $2 r_{a}$ $=119 \mu \mathrm{m}$ for the 103 bubble CRL (CRL 4-1-102). (Adelphi uses the following lens designation: The first digit designates

TABLE II. Measured and calculated parameters of bubble CRLs for APS. 
the "run" or "epoxy batch" in which a lens was made, the second digit is for the number of the lens in that particular batch, and the third number is the number of unit lenses, e.g., 4-1-102 is batch No. 4, CRL No. 1 , and $N=102$. These numbers are primarily for quality control reasons.) Note, for $M$ bubbles there are $M-1$ lenses.

If absorption is neglected, only the central part of a spherical lens approximates the required parabolic shape of an ideal lens. The spherical aberration aperture is defined as the radius $r_{s}$ at which there is $\pi$-phase shift in the electric field at the image plane due to nonparabolic shape of the lens surface. The spherical aberration aperture radius $r_{s}$ is: ${ }^{3}$

$$
r_{s}=\left(4 R^{2} \lambda r_{i}\right)^{1 / 4}=\left(\frac{2 R^{3} \lambda}{\delta N}\right)^{1 / 4}
$$

where $r_{i}$ is the image distance and $\lambda$ is the $\mathrm{x}$-ray wavelength. Rays outside of this aperture do not focus at the same point as those inside. The second half of Eq. (5) is approximately true if $r_{o} \gg f$, an accurate approximation for synchrotron sources, where the distance to the source, $r_{o}$, is quite large.

An important application of the bubble lens would be to increase the intensity of $\mathrm{x}$ rays from a distant source at the image point. Gain is defined as the ratio of the $\mathrm{x}$ ray intensity at the image plane of the lens to the intensity of $x$ rays that would have been obtained at the same plane without a lens. ${ }^{3}$ From energy-conservation arguments, an approximate expression of the gain of a two-dimensional lens is found to be

$$
G \approx \frac{A_{L}}{A_{S}} M^{2} T
$$

where $T$ is the power transmitted through the lens over the incident power impinging on the aperture of the lens (for unit lenses with aperture radii $\approx r_{a}, T \approx 0.43 \mathrm{e}^{-\mu N d}$, where $d$ is the minimum thickness of the unit lens), $A_{L}$ and $A_{S}$ are the area of the lens and of the source, respectively, and $M=r_{o} / f$ is the demagnification. ${ }^{11}$ The spherical aberration aperture $r_{s}$ is not appropriate for determining gain. $A_{L}=\pi r_{a}^{2}$. However, when this aperture is exceeded, the primary effect is a loss of resolution, but the gain is affected much less. Using the Kirchhoff-Huygens equation to determine gain for a spherical lens shows that Eq. (6) is a good approximation. ${ }^{8}$

As can be seen from Eq. (6), the gain is determined by the CRL transmission, $T$, and the square of the demagnification $M$ or $1 / f^{2}$. Thus, the bubble lens with its short focal length and good transmission will give higher gain than most other CRLs. The transmission depends upon the thickness $d$, which in turn depends on the given lens material and on the diameter of the capillary channel. We have fabricated CRLs with minimum thicknesses $d$ as small as $20 \mu \mathrm{m}$.

We used the above formulas to calculate the various parameters of the lenses used here. These calculated values are compared with experimental ones and given in Tables I and II. Minimum lens thickness ( $d$, the minimum distance between spheres) was determined by microscopy.

\section{MEASUREMENTS}

We fabricated ten bubble CRLs and tested them at the Stanford Synchrotron Radiation Laboratory (SSRL) and the

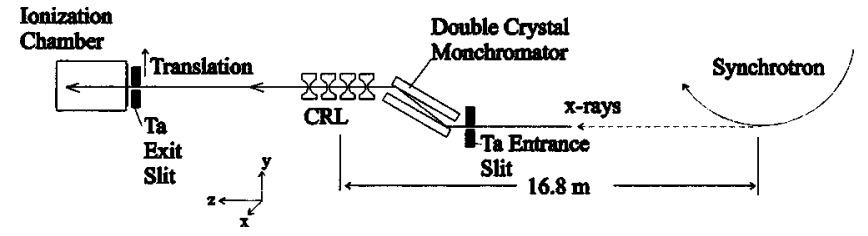

FIG. 2. Experimental apparatus for measuring the profile of a focused x-ray beam and establish the image distance.

Advanced Photon Source (APS). As shown in Fig. 2, essentially the same apparatus was used at these laboratories. Their beamlines possessed double-crystal monochromators $(\mathrm{Si}\langle 111\rangle)$ with $5 \times 10^{-4}$ resolution. At the time of these experiments, these sources had remarkably differing source sizes and source-to-CRL distances. At APS, the source measured $\Delta x=23 \mu \mathrm{m}$ and $\Delta y=97 \mu \mathrm{m}$, and was $58 \mathrm{~m}$ from the CRL. At SSRL, the source measured $\Delta x=0.5 \mathrm{~mm}$ and $\Delta y$ $=2.0 \mathrm{~mm}$, and was $16 \mathrm{~m}$ from the CRL.

For alignment, the CRLs were placed in a goniometer head that could be manually tilted $\theta_{x}$ and $\theta_{y}$. In addition, the CRLs could also be remotely translated orthogonally ( $x$ and $y$ ) to the direction of the x-ray beam to maximize the x-ray transmission though the lens. An x-ray gas ionization detector was placed after a translatable slit for measuring the $\mathrm{x}$-ray beam profile. A Ta slit was adjusted to below $1 \mu \mathrm{m}$ by closing the slits. This method gives a quick and easy profile of the beam and its image distance (and focal length) but lacks the accuracy of other techniques such as translating a knifeedge across the beam.

\section{A. Stanford Synchrotron Radiation Laboratory measurements}

We first used beamline 2-3 at SSRL. The x-ray beam size from this source was approximately $2 \times 20 \mathrm{~mm}^{2}$ at the entrance to the experimental station; however, this size was reduced to approximately $0.2 \times 0.2 \mathrm{~mm}^{2}$ by Ta slits upstream of the monochromator. The $<1 \mu \mathrm{m}$ Ta slit was then translated in the $x$ direction across the focused $\mathrm{x}$-ray beam and its profile obtained. We then manually moved the slits along the $z$ axis of the $\mathrm{x}$-ray beam, measuring its vertical widths by scanning the slits over the beam at each location. Profile of the focal spot size from lens $4-1-102$ for $8 \mathrm{keV}$ is given in Fig. 3(a).

Using these measured widths, we can profile the beam waist as a function of distance from the lens. The full width at half maximum (FWHM) in the vertical direction is plotted as a function of distance from CRL 4-1-102 in Fig. 3(b) for $8 \mathrm{keV}$ photons. This figure shows that the waist of the $\mathrm{x}$-ray beam is converging to minimum at approximately $r_{i}$ $=14 \mathrm{~cm}$. Thus, the source size of $0.44 \mathrm{~mm}$ has been focused to the minimum spot FWHM of $5 \mu \mathrm{m}$, at distance of $14 \mathrm{~cm}$ from the CRL. Thus, the demagnification is $M=.011$. The spot size in the horizontal was measured to be $19 \mu \mathrm{m}$, which is larger because we are imaging a bigger source diameter (1.7 mm, FWHM) in that dimension.

The CRL has an aperture with an absorption profile that has a Gaussian shape that causes stronger absorption of the extreme rays passing through the CRL outer radial regions, in contrast to the better-transmitted rays that pass through the 

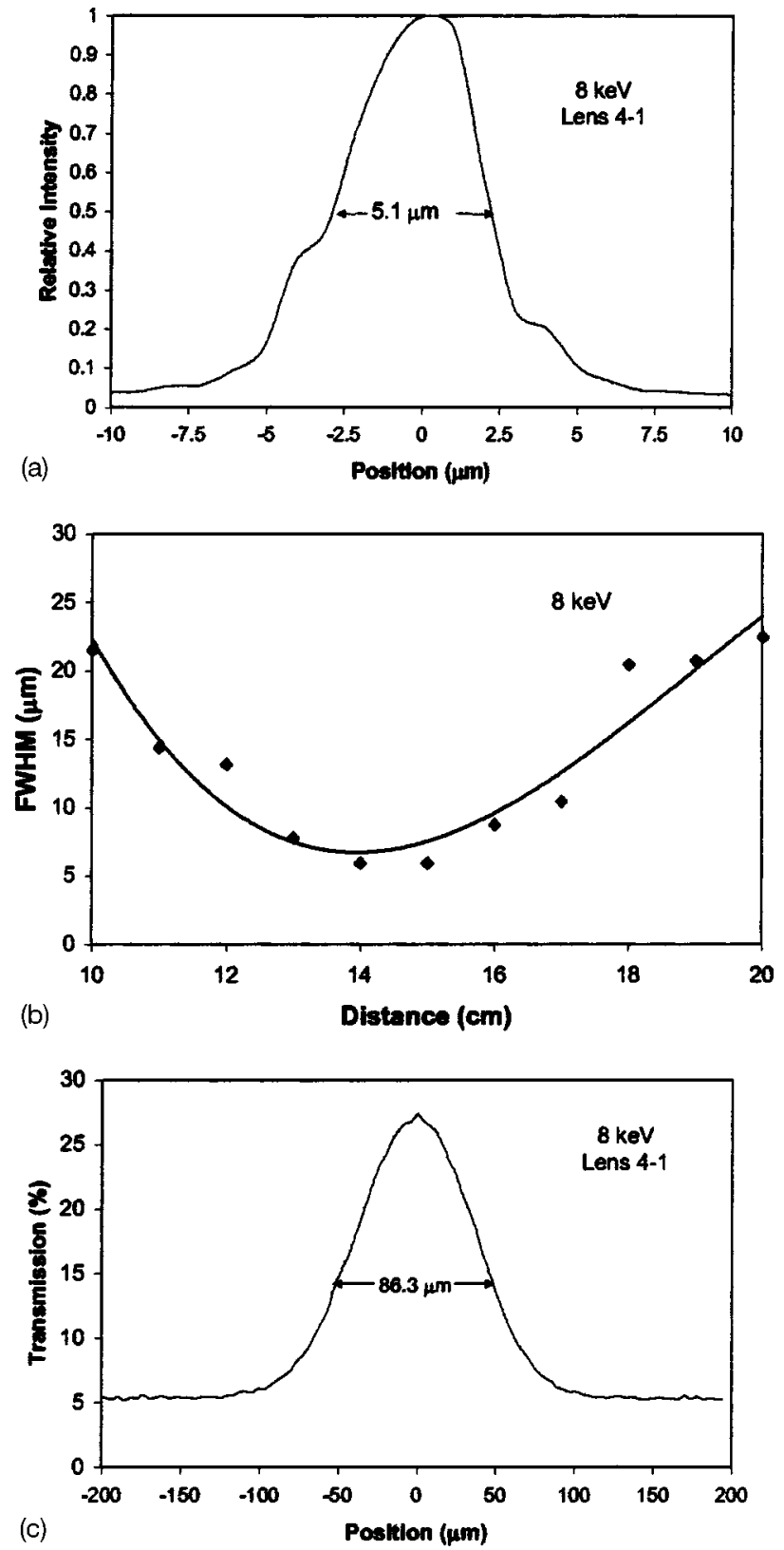

FIG. 3. Characterization of lens 4-1-102: $(2 R=0.2 \mathrm{~mm}, N=102)$. (a) Minimum spot size at $8 \mathrm{keV}$. (b) Profile of the focal spot size as a function of distance. (c) Transmission at $8 \mathrm{keV}$.

central region. We obtained the transmission through the CRLs by narrowing the $\mathrm{x}$-ray beam to $50 \times 50 \mu \mathrm{m}^{2}$ using the upstream Ta slits and translating each CRL through the beam. This gave transmission profiles of the lenses (e.g., 4-1-102 in Fig. 3(c)). The absorption apertures $\left(e^{-2}\right.$ points, not FWHMs) were obtained from these figures and tabulated in Table I. Given the measured transmissions and profiles, we can also determine the gains of these CRLs. The gain values obtained in this fashion varied between 3.5 to 25.5. The calculated and measured parameters for CRL 4-1-102 and other CRLs tested at SSRL are given in Table I.

\section{B. Advanced photon source measurements}

Using the same techniques and much of the same apparatus, we used beamline 5BM-D-DND at APS to profile the
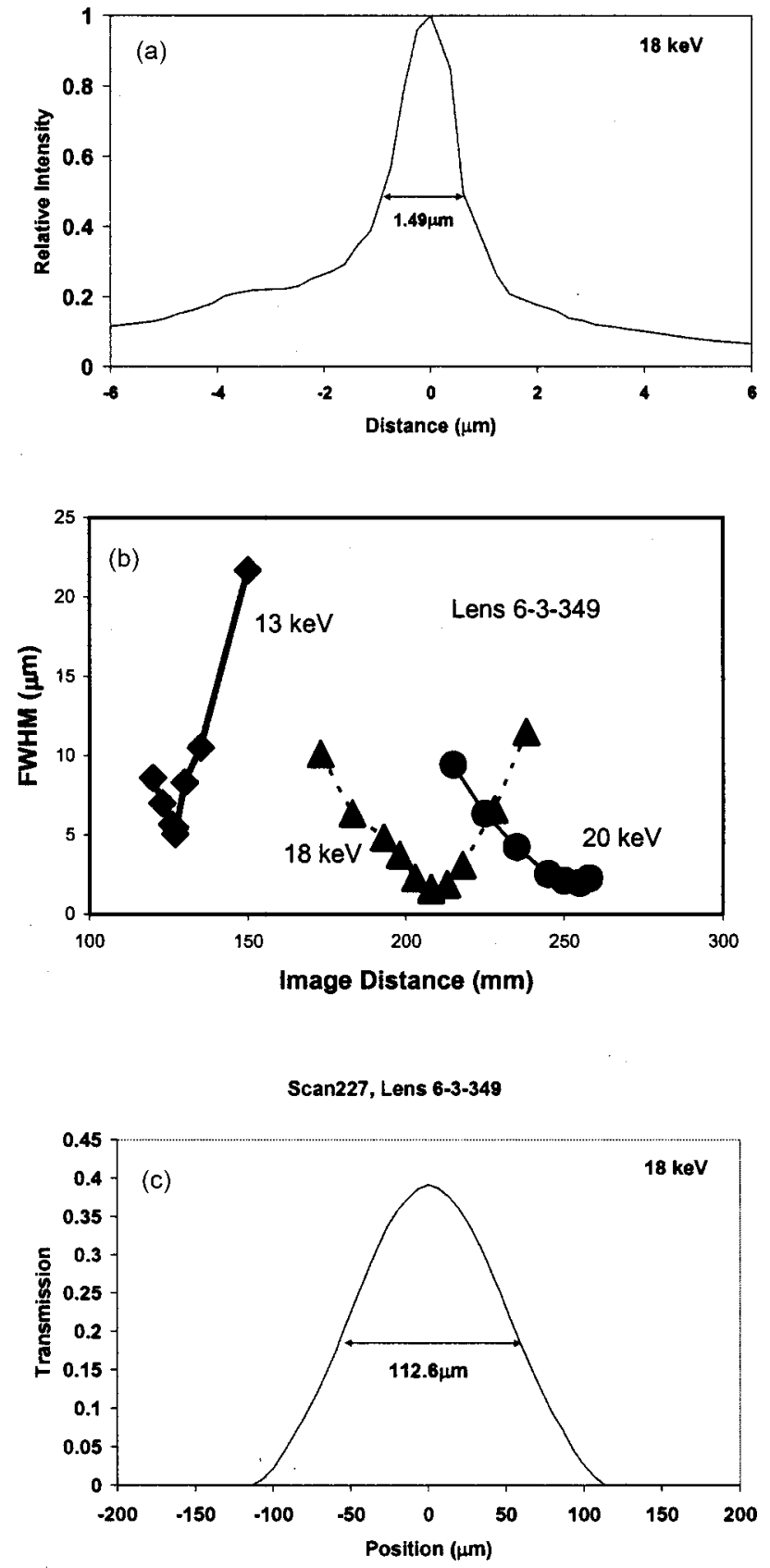

FIG. 4. Characterization of lens 6-3-349 $(2 R=0.2 \mathrm{~mm}, N=349$ lenses): (a) Minimum spot size at $20.8 \mathrm{~cm}$ for $18 \mathrm{keV}$, (b) FWHM of the focal spot size as a function of distance for three energies 13, 18, and $20 \mathrm{keV}$, and (c) transmission for $18 \mathrm{keV}$.

focused x-ray beam using another series of CRLs at higher photon energies and smaller source size. As before, the x-ray beam size was reduced to approximately $180 \times 180 \mu \mathrm{m}^{2}$ by Ta slits in front of the bubble lens. The downstream $<1 \mu \mathrm{m}$ Ta slit was then translated in the $x$ direction across the focused x-ray beam and its profile obtained. For example, the minimum spot-size profile is given for CRL 6-3-349 in Fig. 4(a). The FWHM is $1.5 \mu \mathrm{m}$. CRL 6-3-349 has 349 bubbles in a capillary with a $200 \mu \mathrm{m}$ inside diameter (see Table II).

We then remotely moved the slits along the $z$ axis of the $\mathrm{x}$-ray beam, measuring its vertical widths by scanning the slits over the beam at each location. Profiles of the focal spot size from lens 6-3-349 for 13, 18, and $20 \mathrm{keV}$ are given in 


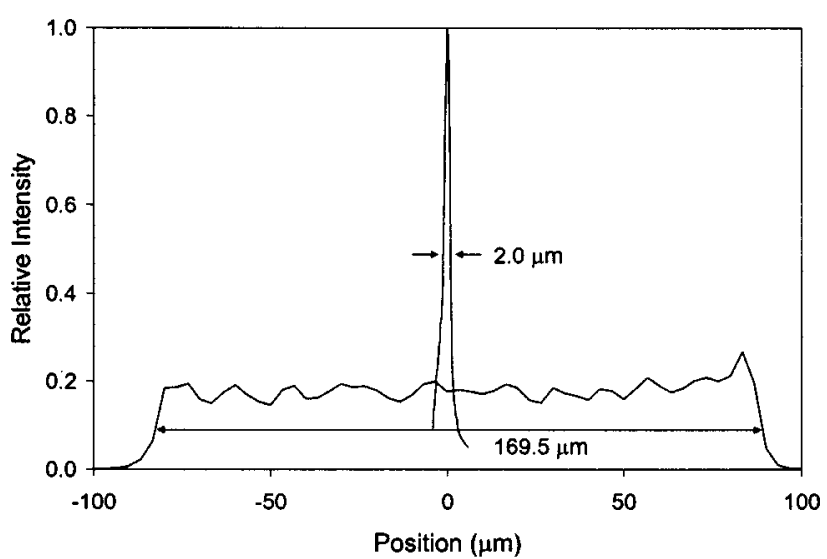

FIG. 5. Beam profile of focused spot and incoming beam in vertical plane for Lens 6-2-300. Note, the beam profiles were taken by the $>1 \mu \mathrm{m}$ slit $x$ direction. The length of the slit was much larger than the lens diameter $(>200 \mu \mathrm{m})$.

Fig. 4(b). The minimum waists of 5, 2.1, and $1.5 \mu \mathrm{ms}$ are seen to be at distances, $r_{i}$, of 13,21 , and $25 \mathrm{~cm}$, respectively.

As before, we obtained the transmission through the CRLs by narrowing the x-ray beam to $50 \times 50 \mu \mathrm{m}^{2}$ using the upstream Ta slits and translating each CRL through the beam. This gave transmission profiles of the CRLs as shown in Fig. 4(c). The absorption apertures ( $e^{-2}$ points, not FWHMs) were obtained from these figures and tabulated in Table I. The calculated and measured parameters for CRL 6-3-349 and other CRLs tested at APS are given in Table II.

The gain was estimated by scanning the $>1 \mu \mathrm{m}$ slit across the x-ray beam at the focus and at the entrance to the CRL. The beam profiles of the focused spot and incoming beam in vertical plane for CRL 6-2-300 were thus obtained at $15 \mathrm{keV}$ (see Fig. 5). The length of the slit was much larger than the lens diameter $(>200 \mu \mathrm{m})$. To obtain a rough idea of the gain, we assume that the spot size in the $y$ direction is the same for both the focused and entrance beams. Taking the ratios of these intensities and squaring the value, we obtain the gain of 25. Other values obtained in this fashion are given in Table II.

\section{DISCUSSION}

CRLs have been made of bubbles embedded in epoxy with focal lengths between 9.6 and $53 \mathrm{~cm}$ for photon energies ranging between 7 to $20 \mathrm{keV}$ with peak transmissions of up to $46 \%$. These lenses give shorter focal lengths than previous methods. The use of the CRLs at these photon energies can have greater application in synchrotron and pro- posed coherent $\mathrm{x}$-ray sources. ${ }^{18}$ The amount of absorption can be further reduced by decreasing the wall thickness, $d$, For photon energies above $30 \mathrm{keV}$, epoxy lenses can achieve a performance comparable to those made of $\mathrm{Be}$ or $\mathrm{Li}$, currently regarded as the best materials. ${ }^{11,12}$ Thus, one can expect CRLs made of epoxy to have an important future in x-ray optics.

\section{ACKNOWLEDGMENTS}

This work was supported by the Byelorussian Foundation of Fundamental Investigations (Grant No. F03MC-005), by the United States National Science Foundation under the Small Business Innovative Research Program (Grant No. DMI-0319668) and was performed at the Stanford Synchrotron Radiation Laboratory and the Argonne National Laboratory, which are operated by the Department of Energy, Office of Basic Energy Sciences.

${ }^{1}$ F. L. Pedrotti and L. S. Pedrotti, Introduction to Optics (Prentice-Hall, Englewood Cliffs, NJ, 1987), Chap. 4.

${ }^{2}$ T. Tomie, U.S. Patent No. 5,594,773 (14 Jan. 1997) Foreign Appl. Date Feb. 18, 1994, Japan No. 6-45288.

${ }^{3}$ A. Snigirev, V. Kohn, I. Snigireva, and B. Lengeler, Nature (London) 384, 49 (1996).

${ }^{4}$ J. T. Cremer, M. A. Piestrup, H. R. Beguiristain, C. K. Gary, R. H. Pantell, and R. Tatchyn, Rev. Sci. Instrum. 70, 3545 (1999).

${ }^{5}$ B. Lengeler, J. Tommler, A. Snigirev, I. Snigireva, and C. Raven, J. Appl. Phys. 84, 5855 (1998).

${ }^{6}$ B. Lengeler, C. G. Schroer, M. Richwin, J. Tümmeler, M. Drakopolulos, A. Snigirev, and I. Snigireva, Appl. Phys. Lett. 74, 3924 (1999).

${ }^{7}$ M. A. Piestrup, R. H. Pantell, J. T. Cremer, and H. R. Beguiristain, U.S. Patent, 6,269,145 B1 (Jul. 31, 2001).

${ }^{8}$ R. H. Pantell, J. Feinstein, M. A. Piestrup, H. R. Beguiristain, C. K. Gary, and J. T. Cremer, Rev. Sci. Instrum. 72, 48 (2001).

${ }^{9}$ M. A. Piestrup, H. R. Beguiristain, C. K. Gary, J. T. Cremer, R. H. Pantell, and R. Tatchyn, Nucl. Instrum. Methods Phys. Res. B 173, 170 (2001).

${ }^{10}$ M. A. Piestrup, H. R. Beguiristain, C. K. Gary, J. T. Cremer, and R. H. Pantell, Rev. Sci. Instrum. 71, 4375 (2000).

${ }^{11}$ H. R. Beguiristain, J. T. Cremer, M. A. Piestrup, C. K. Gary, and R. H. Pantell, Opt. Lett. 27, 778 (2002).

${ }^{12}$ J. T. Cremer, M. A. Piestrup, H. R. Beguiristain, C. K. Gary, and R. H. Pantell, Rev. Sci. Instrum. 74, 2262 (2003).

${ }^{13}$ Y. I. Dudchik and N. N. Kolchevsky, Nucl. Instrum. Methods Phys. Res. A 421, 361 (1999).

${ }^{14}$ Y. I. Dudchik, N. N. Kolchevsky, F. F. Komarov, Y. Kohmura, M. Awaji, Y. Suzuki, and T. Ishikawa, Nucl. Instrum. Methods Phys. Res. A 454, $512(2000)$.

${ }^{15}$ Y. I. Dudchik and N. N. Kolchevsky, Proc. SPIE 4145, 235 (2001).

${ }^{16}$ Y. Kohmura, M. Awaji, Y. Suzuki, T. Ishikawa, Y. I. Dudchik, N. N. Kolchevsky, and F. F. Komarov, Rev. Sci. Instrum. 70, 4161 (1999).

${ }^{17}$ Y. Kohmura, K. Okada, A. Takeuchi, H. Takano, Y. Suzuki, T. Ishikawa, T. Ohigashi, and H. Yokosuka, Nucl. Instrum. Methods Phys. Res. A 467, 881 (2001).

${ }^{18}$ R. H. Pantell, J. Feinstein, H. R. Beguiristain, M. A. Piestrup, C. K. Gary, and J. T. Cremer, Appl. Opt. 40, 5100 (2001). 